\title{
Antimicrobial susceptibility in thermophilic Campylobacter species isolated from pigs and chickens in South Africa
}

\author{
A Jonker ${ }^{a^{*}}$ and J A Picard ${ }^{a}$
}

\begin{abstract}
Campylobacter jejuni is one of the leading causes of sporadic food-borne bacterial disease in humans. In intensive poultry and pig rearing systems the use of oral antibiotics is essential to maintain health. Consequently, there is a high risk for the thermophilic Campylobacter jejuni and C. coli resident in the intestinal tract of food animals to develop resistance to commonly used antibiotics. Contamination of meat or eggs with pathogenic strains of resistant Campylobacter could, therefore, result in a form of campylobacteriosis in humans that is difficult to treat. The aim of this investigation was to determine the antimicrobial susceptibility of thermophilic Campylobacter spp. isolated from pigs and poultry by the broth microdilution minimum inhibitory concentration (MIC) test. A total of 482 samples from the Western Cape and Gauteng provinces was collected and analysed. Thirty-eight Campylobacter isolates were obtained. Analysis of data revealed that C. jejuni strains mainly of poultry origin were more resistant to the fluoroquinolones, macrolides and tetracyclines and the C. coli strains were more resistant to the macrolides and lincosamides. Multiresistance was also detected in 4 Campylobacter strains from the Western Cape. With the exception of tetracyclines, strains from high health Gauteng broiler farms were susceptible to antibiotics used to treat Campylobacter infections.
\end{abstract}

Keywords: antimicrobial susceptibility, broth microdilution, Campylobacter, minimum inhibitory concentration, thermophilic.

Jonker A, Picard J A Antimicrobial susceptibility in thermophilic Campylobacter species isolated from pigs and chickens in South Africa. Journal of the South African Veterinary Association (2010) 81(4): 228-236 (En.). Department of Veterinary Tropical Diseases, Faculty of Veterinary Science, University of Pretoria, Private Bag X04, Onderstepoort, 0110 South Africa.

\section{INTRODUCTION}

The thermophilic campylobacters Campylobacter jejuni and C. coli occur worldwide as commensals in the digestive tracts of healthy animals, especially birds $^{38}$. Campylobacter jejuni is isolated most commonly from broilers and C. coli from pigs ${ }^{11,42}$.

Although of low virulence in animals, some strains of both these species have the ability to cause potentially serious diarrhoeal illness in humans ${ }^{26}$. In fact, campylobacteriosis is considered to be one of the most common causes of sporadic food-borne bacterial illness worldwide ${ }^{2,16,20}$. Animal-derived foods, especially poultry products, are thought to be the major source of Campylobacter infections in humans ${ }^{2,9,38}$.

Campylobacter jejuni predominates in human infections where it accounts for approximately $95 \%$ of cases campylo-

${ }^{a}$ Department of Veterinary Tropical Diseases, Faculty of Veterinary Science, University of Pretoria, Private Bag X04, Onderstepoort, 0110 South Africa.

*Author for correspondence. Present address: Provincia Veterinary Laboratory, Private Bag X5020, Stellenbosch, 7599 South Africa. E-mail: annelizej@ elsenburg.com Received: March 2010. Accepted: November 2010 bacteriosis. This disease in humans is acute and tends to be self-limiting, but serious complications such as GuillainBarré syndrome may develop in a small number of patients ${ }^{5,38}$.

Macrolides such as erythromycin, azithromycin and clarithromycin are preferred in human cases that require medication $^{2,3}$. Furthermore, C. coli and C. jejuni are also susceptible to the aminoglycosides chloramphenicol, clindamycin, nitrofurans and imipenem. Varying rates of resistance have been recorded in several countries to tetracyclines, erythromycin, fluoroquinolones, the beta-lactams and metronidazole e,24,28,41. $^{\text {. }}$ Intrinsic resistance to vancomycin, rifampin, trimethoprim ${ }^{3}$, bacitracin and novobiocin exists ${ }^{39}$

Three minimum inhibitory concentration methods have been validated to test the susceptibility of thermophilic campylobacters to antibiotics, namely, broth microdilution, agar dilution and the epsilometer (E-test) tests ${ }^{28}$. Both the broth microdilution and agar dilution are recommended by the Clinical and Laboratory Standards Institute (CLSI) ${ }^{7}$.
The primary aim of this investigation was to isolate and determine the antimicrobial susceptibility patterns of thermophilic Campylobacter spp. isolated from the intestinal tract of poultry and pigs, both important food animals that are known to have a high carriage of these intestinal bacteria ${ }^{5,12,24,36}$.

\section{MATERIALS AND METHODS}

\section{Collection and culture}

During November 2007 to June 2008, 226 intact chicken caecae and $25610 \mathrm{~cm}$ lengths of porcine colons were collected during necropsy for a non-enteric disease and from healthy animals at abattoirs. These samples were placed individually in sterile plastic containers, sealed and transported on ice without preservatives or transport media to the Western Cape Provincial Laboratory or Department of Veterinary Tropical Diseases, Faculty of Veterinary Science, Pretoria, where they were cultured within 3 hours of collection.

To improve the sensitivity of isolation, 2 methods were used. In the 1st method the intestinal mucosa was rubbed with a cotton-tipped swab. This swab was used to inoculate a plate of Skirrow's agar (SA) (CM0935 \& SR 0069, Oxoid Ltd). In the second method a cellulose nitrate filter with pore size $0.65 \mu \mathrm{m}$ (Sartorius Stedim Biotech) was placed on a plate of Columbia agar (CM0331, Oxoid Ltd) containing $5 \%$ defribrinated sheep's blood (BCA) and a generous sample (approximately $0.5 \mathrm{ml}$ ) of intestinal content placed on $\mathrm{it}^{33}$. The plates were incubated in Campylobacter gas (CampyGen) (CN0225, Oxoid Ltd) at $42{ }^{\circ} \mathrm{C}$ for 48 to 72 hours. After 24 hours incubation the filter on the BCA was removed, the inoculum streaked out to obtain single colonies of bacteria and re-incubated under the same conditions as previously.

Any small, dew-like colonies isolated were identified as Campylobacter-like if they comprised of Gram-negative curved bacteria that were catalase- and oxidasepositive. Such colonies were then identified to species level by means of biochemical analyses $^{33}$. After identification and follow- 
Table 1: Potencies, final amount of powder, solvents, diluents and volume of diluents.

\begin{tabular}{|c|c|c|c|c|c|}
\hline Antibiotic & $\begin{array}{r}\text { Potency } \\
(\mu \mathrm{g} / \mathrm{mg})\end{array}$ & $\begin{array}{l}\text { Amount of powder } \\
(\mathrm{mg})\end{array}$ & Solvent & Diluent & $\begin{array}{l}\text { Volume of diluent } \\
(\mathrm{m} \ell)\end{array}$ \\
\hline Chlortetracycline & 852 & 160 & Water & Water & 106.5 \\
\hline Doxycycline & 847 & 180 & Water & Water & 119.1 \\
\hline Enrofloxacin & 996 & 150 & $\begin{array}{l}1 / 2 \text { volume water, then add } \\
1 \mathrm{~mol} / \mathrm{L} \mathrm{NaOH} \text { dropwise to } \\
\text { dissolve }\end{array}$ & Water & 116.7 \\
\hline Erythromycin & 655 & 210 & 95 \% Ethanol & Water & 107.5 \\
\hline Fosfomycin & 761 & 170 & $95 \%$ Ethanol & Water & 101.1 \\
\hline Lincomycin & 786 & 160 & Water & Water & 98.3 \\
\hline Norfloxacin & 998 & 130 & $\begin{array}{l}1 / 2 \text { volume water, then add } \\
1 \mathrm{~mol} / \mathrm{L} \mathrm{NaOH} \text { dropwise to } \\
\text { dissolve }\end{array}$ & Water & 101.4 \\
\hline Tiamulin & 986 & 170 & Water & Water & 105.7 \\
\hline Tylosin & 978 & 170 & $95 \%$ Ethanol & Water & 129.9 \\
\hline
\end{tabular}

ing the manufacturer's instructions, isolates were frozen on Microbank ${ }^{\circledR}$ beads (Prolab Diagnostics) at $-70^{\circ} \mathrm{C}$ in an ultralow-temperature freezer (New Brunswick Scientific).

\section{Minimum inhibitory concentration tests}

Susceptibility of isolates to a selection of commonly used veterinary antimicrobial drugs in poultry and pigs was determined by broth microdilution as recommended by the CLSI ${ }^{7}$. For the testing of Western Cape isolates, stock solutions of the following analytical grade antimicrobial drugs were made: chlortetracycline (Fujian Fukang Pharmaceutical Co. Ltd, China), doxycycline (Yancheng Suhai Pharmaceutical Co. China), enrofloxacin (Kirsch Pharma, South Africa), erythromycin (Ercros Industrial, South Africa), fosfomycin (Hangzhou Chyszem Biotech Co. Ltd, China), lincomycin (Nanyang Pukang Pharmaceutical Co. Ltd, China), norfloxacin (Dankong Industry \& Trade Group, Co. Ltd, China), tiamulin (Shandong Lukang Shelile, China) and tylosin (Biesterfeld, Germany).

Isolates from poultry farms in Gauteng were tested using a commercial MIC test (Trek Sensititre Bovine/Porcine plate format BOP06F, Trek Diagnostic Systems, Separation Scientific) which contained ceftiofur, gentamicin, neomycin, spectinomycin, florfenicol, chlortetracycline, oxytetracycline, penicillin, ampicillin, enrofloxacin, danofloxacin, tiamulin, tylosin, tulathromycin, tilmicosin and lincomycin.

Methodologies differ between the laboratories in the Western Cape and Gauteng, because the most conveniently available methods were used.

\section{Preparation of MIC panels}

Stock solutions of antimicrobials (refer to Table 1 for potency, amount of powder weighed and solvents) were prepared, aliquotted and frozen ${ }^{7}$. The stock solutions were defrosted and diluted 1:10 in cation-adjusted Mueller Hinton broth (CAMB) (CM 0405, Oxoid Ltd) to obtain working dilutions. The working dilutions were added to the first column of wells on a 96-well ' $U$ '-bottomed microtitre plate and diluted in serial 2-fold dilutions using cation-adjusted Mueller-Hinton broth (CAMHB) as the diluent (CM 0405, Oxoid Ltd). The dilution range included quality control ranges as well as any available breakpoints. One well was used as a growth control and received $100 \mu \ell$ of CAMHB only. Plates were prepared the day before testing and stored in a refrigerator.

\section{Antimicrobial susceptibility testing}

Microbank beads containing frozen isolates were streaked on BCA without antibiotics. The plates were incubated in CampyGen at $42^{\circ} \mathrm{C}$ for 48 hours. Two subsequent subcultures were made and incubated in CampyGen at $42{ }^{\circ} \mathrm{C}$ for 48 hours ${ }^{10}$. One full loop of culture was picked from 48-hour old cultures and suspended in 2 m of $0.9 \%$ saline to obtain a turbidity approximately equal to a 0.5 McFarland standard. This suspension was initially diluted 1:100 in CAMHB to obtain the final inoculum of approximately $10^{5}$ colony forming units $(\mathrm{cfu} / \mathrm{m} \ell)^{5,10}$. However, as the Campylobacter field strains yielded hardly any visible growth in CAMHB and very few colonies on the purity control plate, a decision was made to dilute the initial suspension 1:50 to obtain the final inoculum of approximately $2 \times 10^{5} \mathrm{cfu} / \mathrm{m} \ell$.

One hundred microlitre volumes of inoculum were pipetted into each well of the testing panels and the plates were covered with a lid. The inoculated panels were incubated microaerophilically at $37^{\circ} \mathrm{C}$ for 48 hours, after which the panels were read ${ }^{7,10}$.
Quality control procedures simultaneously performed with each batch of tests were as follows. Batch control using reference strains (Escherichia coli ATCC 25922 (American Type Culture Collection, USA), Enterococcus faecalis ATCC 29212, Staphylococcus aureus ATCC 29213 and Campylobacter jejuni ATCC 33560). An inoculum density/purity control was performed for each isolate and a growth control well was carried out on each plate (Table 2). The goal of quality control was to monitor the precision and accuracy of the test as well as the performance of reagents, viability of organisms and the performance of persons carrying out the tests and interpreting results ${ }^{8}$.

\section{Statistics}

Descriptive statistics were predominantly used to perform inter-host, interprovincial and inter-species comparisons. These included determining the percentage resistance using published breakpoint values ${ }^{1,5,19}$ for the tested antibiotics, the $\mathrm{MIC}_{50}$ (equivalent to the median value) and $\mathrm{MIC}_{90}$, as well as the distribution percentages of the MICs. Using an internet calculator (http://faculty. vassar.edu/lowry/utest.html), the MannWhitney $U$-test was used to determine whether there were any statistical differences $^{25}$. The non-parametric MannWhitney $U$-test (synonym Wilcoxon rank-sum test ${ }^{27}$, was selected as it is best suited to compare 2 sets of independent data that do not have a normal distribution.

\section{RESULTS}

\section{Isolation and identification}

Three hundred and sixty-two samples were obtained from pigs $(n=256)$ and chickens $(n=106)$ originating from a total of 24 farms in the Western Cape Province. Thirteen farms were piggeries and 11 were poultry farms. A total of 120 caeca 
Table 2: MIC Breakpoints and quality control ranges in $\mu \mathrm{g} / \mathrm{m} \ell$.

\begin{tabular}{|c|c|c|c|c|c|c|}
\hline Antibiotic & Resistant & Susceptible & $\begin{array}{c}\text { Escherichia } \\
\text { coli } \\
\text { ATCC } 25922 \\
37^{\circ} \mathrm{C} / 24 \text { hours }\end{array}$ & $\begin{array}{c}\text { Staphylococcus } \\
\text { aureus } \\
\text { ATCC } 29213 \\
37^{\circ} \mathrm{C} / 24 \text { hours }\end{array}$ & $\begin{array}{c}\text { Enterococcus } \\
\text { faecalis } \\
\text { ATCC } 29212 \\
37^{\circ} \mathrm{C} / 24 \text { hours }\end{array}$ & $\begin{array}{c}\text { Campylobacter } \\
\text { jejuni } \\
\text { ATCC } 33560 \\
37^{\circ} \mathrm{C} / 48 \text { hours }\end{array}$ \\
\hline Doxycycline & $\geq 8$ & $\leq 4$ & $0.5-2$ & $0.12-1$ & $8-32$ & $0.12-0.5$ \\
\hline Enrofloxacin & $\geq 4$ & $\leq 1$ & $0.008-0.03$ & $0.03-0.12$ & $0.12-1$ & - \\
\hline Erythromycin & $\geq 8$ & $\leq 1$ & $-^{\dagger}$ & $0.25-1$ & $1-4$ & $0.5-2$ \\
\hline Tetracycline & $\geq 8$ & $\leq 4$ & $0.5-2$ & $0.12-1$ & $8-32$ & $0.25-2$ \\
\hline Tiamulin & $\geq 1^{*}$ & $\leq 0.1$ & - & $0.5-2$ & - & - \\
\hline Tylosin & $\geq 64^{\#}$ & $\leq 32$ & - & $0.5-4$ & $0.5-4$ & - \\
\hline Tilmicosin & $\geq 32$ & $\leq 16$ & - & $1-4$ & $8-32$ & - \\
\hline Tulathromycin & $\geq 64$ & $\leq 16$ & - & $2-8$ & $4-32$ & - \\
\hline Fosfomycin & $\geq 128^{* *}$ & $\leq 128$ & - & - & - & - \\
\hline Lincomycin/Clindamycin & $\geq 4$ & $\leq 0.5$ & - & $0.06-0.025$ & $4-16$ & $0.12-1$ \\
\hline Gentamicin & $\geq 8$ & $\leq 4$ & $0.25-1$ & $0.1-2$ & $4-6$ & $0.5-2$ \\
\hline Spectinomycin & $\geq 128$ & $\leq 64$ & $8-64$ & $64-256$ & $64-256$ & - \\
\hline Penicillin & $\geq 16$ & $\leq 8$ & - & $0.25-1$ & $1-4$ & - \\
\hline Ampicillin & $\geq 16$ & $\leq 8$ & $2-8$ & $0.5-2$ & $0.5-2$ & - \\
\hline Ceftiofur & $\geq 8$ & $\leq 2$ & $0.25-1$ & $0.25-1$ & - & - \\
\hline Florfenicol & $\geq 32$ & $\leq 8$ & $2-8$ & $2-8$ & $2-8$ & $1-4$ \\
\hline
\end{tabular}

Adapted from Antibiogram Committee of the French Society for Microbiology (1999, cited by Avrain et al. ${ }^{5} ; \mathrm{CLSI}^{7}$ ).

${ }^{\dagger} \mathrm{A}$ dash indicates that no acceptable range has been established.

${ }^{*}, \#,{ }^{* *}=$ Refs 19,1 and 4 , respectively.

was also collected from 6 poultry farms in Gauteng Province.

Specimens from 13 of the 30 farms (43\%) sampled yielded thermophilic Campylobacter isolates. Five of these farms were piggeries and 8 were poultry farms. Thirty-eight Campylobacter isolates were obtained from the 482 samples $(7.88 \%)$, 6 from pigs and 32 from chickens.

Based on the hippurate hydrolysis test $^{33}, 24$ of the isolates were identified as C. jejuni and the other 14 isolates as C. coli. Of the 6 isolates from pigs, 1 was $C$. jejuni and 5 were $C$. coli. Of the 32 isolates from chickens, 23 were C. jejuni and 9 were C. coli. Sixty-five per cent of isolates were obtained from carcasses presented for necropsy. The distribution of the cultured Campylobacter spp. is shown in Table 3.

\section{Antimicrobial susceptibility testing results}

The $\mathrm{MIC}_{50}$ and $\mathrm{MIC}_{90}$ are minimum inhibitory concentrations of an antimicrobial at which growth of $50 \%$ and $90 \%$ of isolates respectively, are inhibited ${ }^{24}$. The $\mathrm{MIC}_{50}$ and $\mathrm{MIC}_{90}$ values were higher from Campylobacter spp. isolated in

Table 3: A summary of Campylobacter jejuni and $C$. coli cultured from the intestinal tract of healthy broilers and pigs.

\begin{tabular}{|c|c|c|c|c|c|}
\hline & \multicolumn{2}{|c|}{ Porcine } & \multicolumn{2}{|c|}{ Poultry } & \multirow[t]{2}{*}{ Total } \\
\hline & C. jejuni & C. coli & C. jejuni & C. coli & \\
\hline Western Cape & 1 & 5 & 4 & 6 & 16 \\
\hline Gauteng & 0 & 0 & 18 & 4 & 22 \\
\hline Total & 1 & 5 & 22 & 10 & 38 \\
\hline
\end{tabular}

the Western Cape Province than those from Gauteng Province (for those that could be compared.). Campylobacter spp. from the Western Cape, as revealed in Figs 1 and 2, and with the exception of tiamulin resistance, tended to be divided into resistant and non-resistant populations. This was not observed in isolates from poultry samples from Gauteng, where resistance to antimicrobials was limited to the tetracyclines $(95.5 \%), \beta$-lactams $(95.4 \%)$, ceftiofur $(95.5 \%)$ and ampicillin $(85.5 \%)$. Campylobacter spp. isolated from the Western Cape, had a lower percentage of resistance to the tetracyclines $(57.7 \%)$, which is considered to be statistically significant $(P=<0.0001)$, and a higher level of resistance to enrofloxacin $(P=0.0392)$, macrolides $(P=$ $0.0262)$ and lincosamides $(P=0.0001)$. There was also a tendency for these bacteria to be more resistant to the pleuromutilins (tiamulin) $(P=0.0985)$.

When $\mathrm{MIC}_{50}$ and $\mathrm{MIC}_{90}$ values, and the percentage distribution graphs were compared (Tables 4, 5 and 6), it was revealed that $C$. coli, the predominant isolate from pigs, tended to be, with the exception of resistance to the lincosamides (lincomycin) and macrolides, more susceptible to antimicrobials than C. jejuni. The Western Cape C. coli isolates yielded a $\mathrm{MIC}_{50}$ and $\mathrm{MIC}_{90}$ of $>43 \mu \mathrm{g} / \mathrm{m}$ l to erythromycin and a MIC $_{90}$ of $>43 \mu \mathrm{g} / \mathrm{m} \ell$ to the lincosamides and tylosin. In Fig. 1 this is illustrated by the high peak at the $>43 \mu \mathrm{g} / \mathrm{m} l$ category for the C. coli group. Consequently there was a tendency for $C$. coli to be more resistant than $C$. jejuni to the macrolides, erythromycin $(P=0.0708)$ and tylosin $(P=0.063)$. However, the 4 C. coli isolated from broilers on a farm in Gauteng, unlike those from the Western Cape, were highly susceptible to the lincosamides and macrolides. The $C$. coli were considered to be more susceptible than the $C$. jejuni to the tetracyclines chlortetracycline $(P=0.0307)$ and doxycycline $(P=$ 0.0446).

Interestingly only the thermophilic Campylobacter spp. originating from the Western Cape revealed any resistance to the fluoroquinolones, $33.65 \%$ in the case of enrofloxacin and $43.65 \%$ in the case of norfloxacin.

Four of the $16(25 \%)$ isolates (3 C. coli and 1 C. jejuni) from the Western Cape were resistant to 3 or more antibiotic classes, including the tetracyclines, macrolides, lincosamides, pleuromutulins and fluoroquinolones. No multi-resistant Campylobacter spp. were isolated from the flocks in Gauteng.

Unusually, 4 C. jejuni isolates and 1 C. coli isolate from the Western Cape (all from poultry) were nalidixic acid resistant 


\section{A) Chlortetracycline}

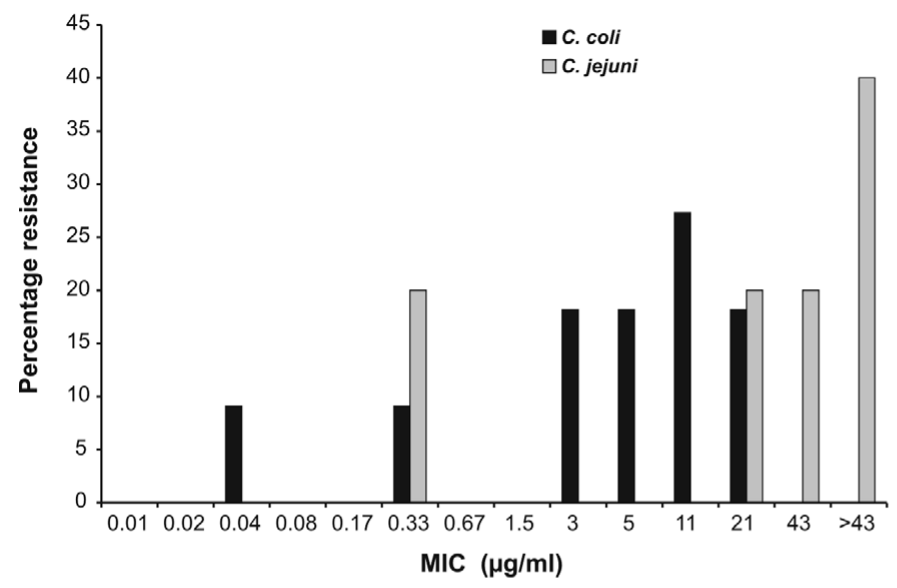

C) Erythromycin

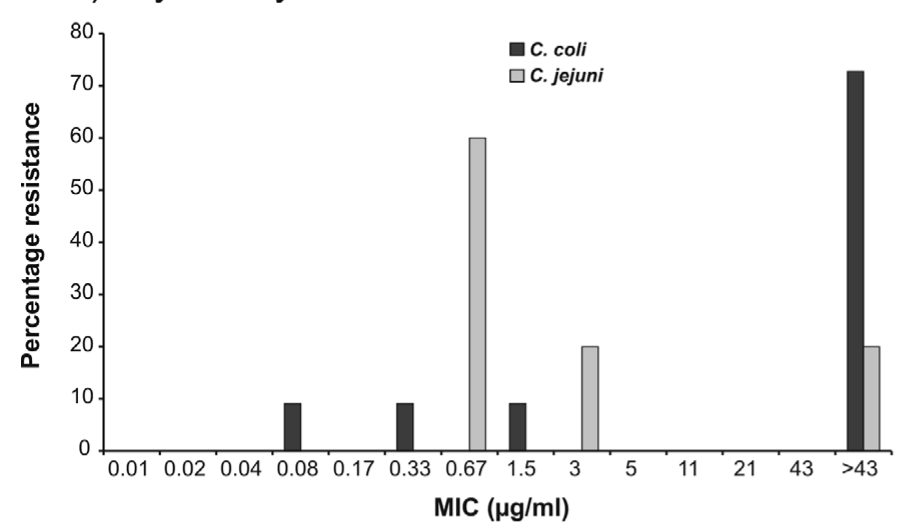

E) Lincomycin

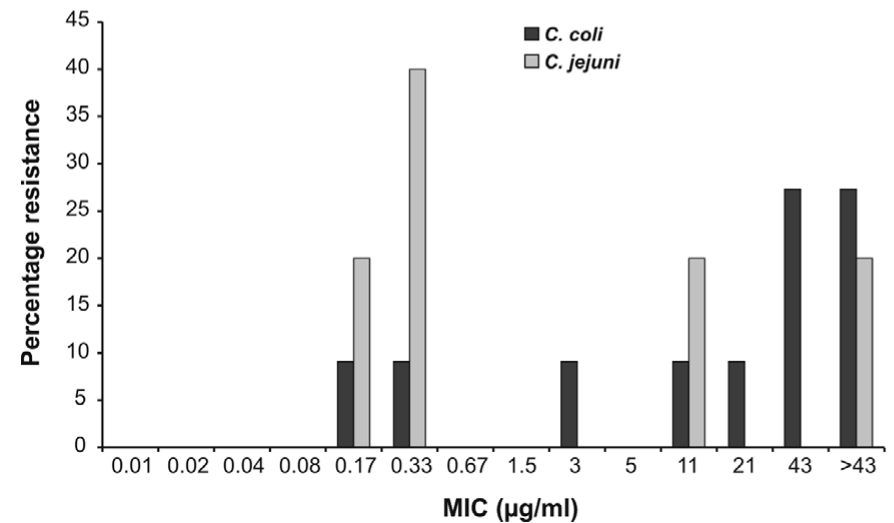

B) Doxycycline
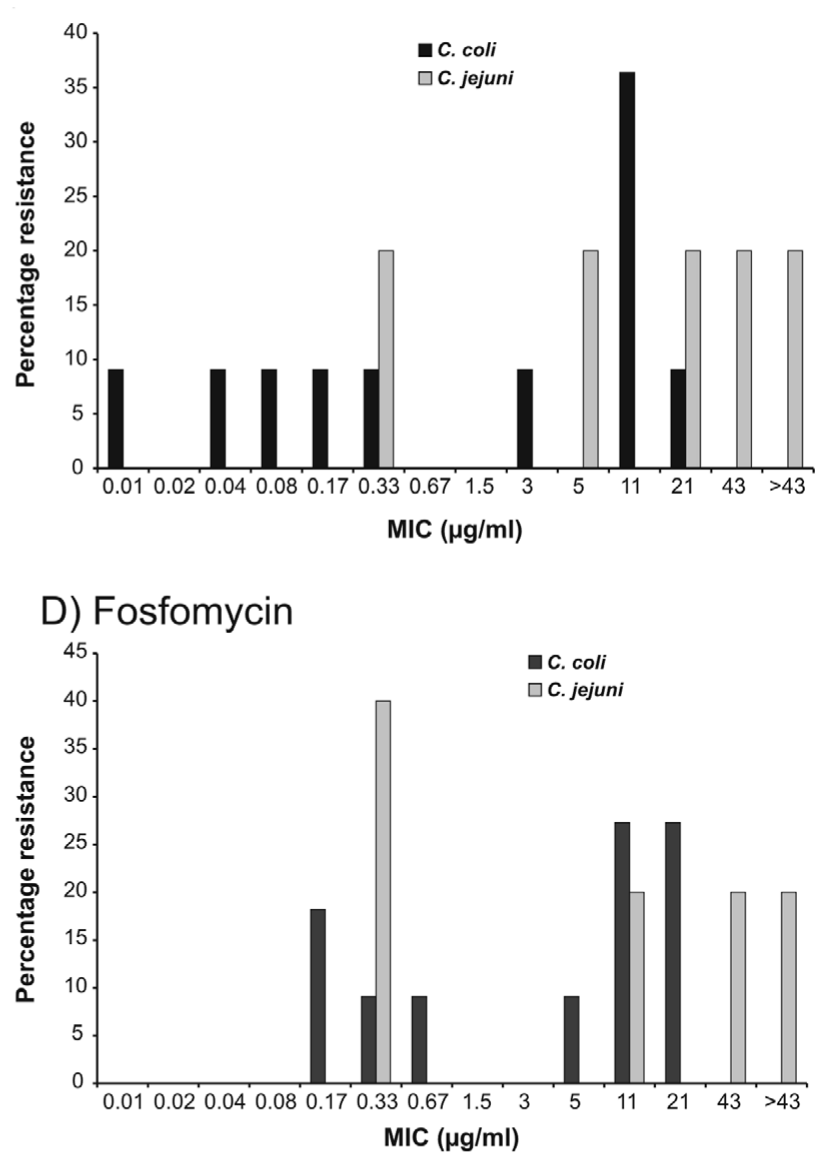

F) Enrofloxacin

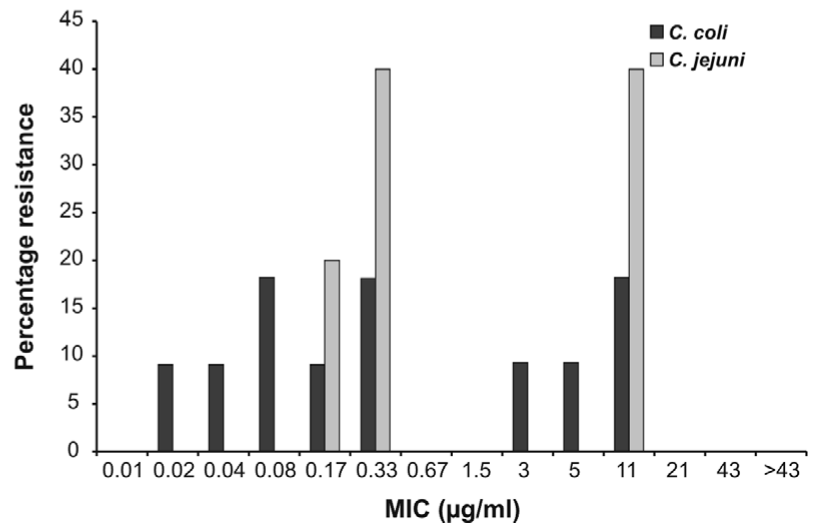

Fig. 1: Percentage distribution of MIC values to the indicated antibiotics of Campylobacter jejuni $(n=5)$ and $C$. coli $(n=11)$ isolated from the Western Cape.

on the disk diffusion sensitivity test. Three of these isolates had MICs of $11 \mu \mathrm{g} / \mathrm{m} \ell$ for enrofloxacin and 3 had MICs of $\geq 11 \mu \mathrm{g} / \mathrm{m} \ell$ for norfloxacin. Two isolates had MICs of $\geq 11 \mu \mathrm{g} / \mathrm{m} \ell$ for both antibiotics.

As pigs are given different therapeutic regimens from those of poultry, it was also decided to determine whether there were any differences between the campylobacters of porcine and those of poultry origin. Porcine Campylobacter strains were considerably more susceptible to tetracyclines (percentage resistance
$34.4 \%$ and $33.3 \%$ to chlortetracycline and doxycycline, respectively) than the poultry strains $(70 \%$ and $60 \%$ percentage resistance to chlortetracycline and doxycycline, respectively). However, these differences were not statistically significant when the MIC values were compared (chlortetracycline $P=0.2389$ and doxycycline $P=0.1922$ ). The thermophilic campylobacters of poultry origin were more resistant to enrofloxacin $(P=$ $0.0021)$ and tended to be resistant to norfloxacin $(P=0.0793)$. Even though not statistically significant, a higher percent- age of porcine strains were resistant to the lincosamides $(83.3 \%)$ and erythromycin $(66.7 \%)$.

\section{DISCUSSION}

Worldwide, most poultry flocks are considered to be the natural hosts of C. jejuni with prevalence rates from 10 to $82 \%$ in conventionally reared positive flocks and an even higher prevalence in free-range chickens (54\% to $100 \%)^{18}$. Similarly, the prevalence of Campylobacter spp. may be as high as $100 \%$ in piggeries, the only difference being that $C$. coli tends 
G) Norfloxacin

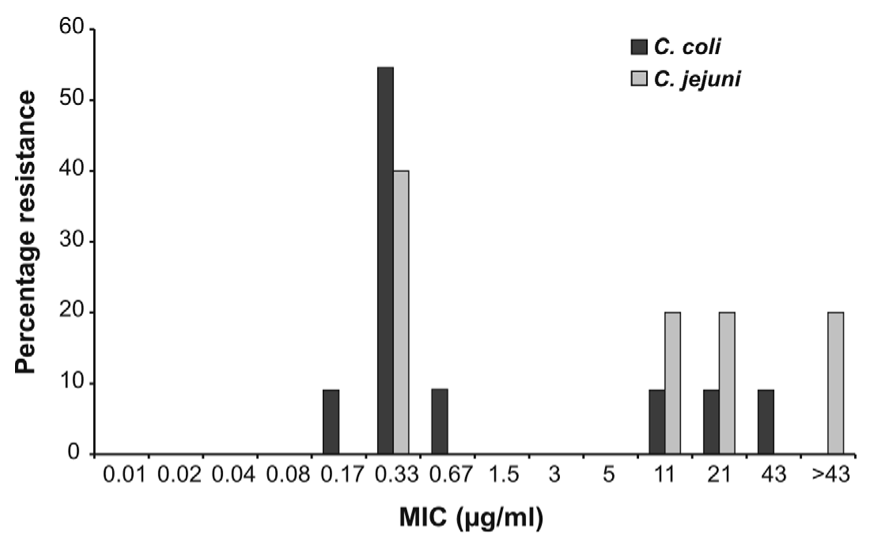

H) Tiamulin

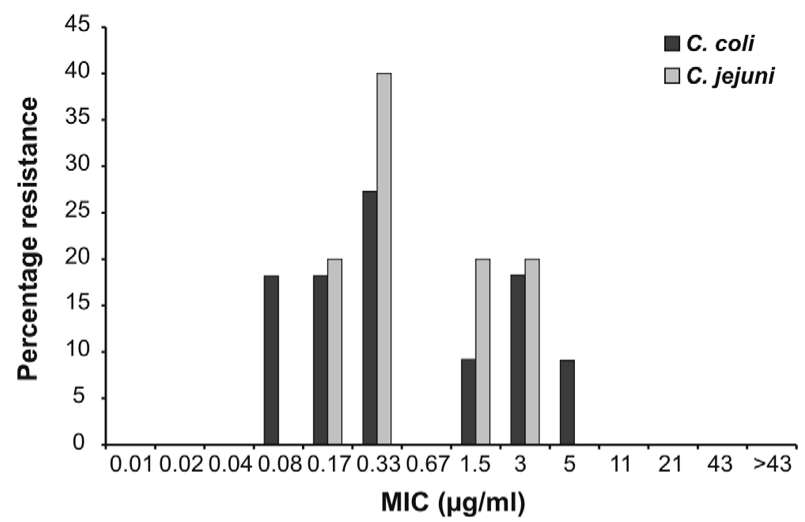

\section{I) Tylosin}

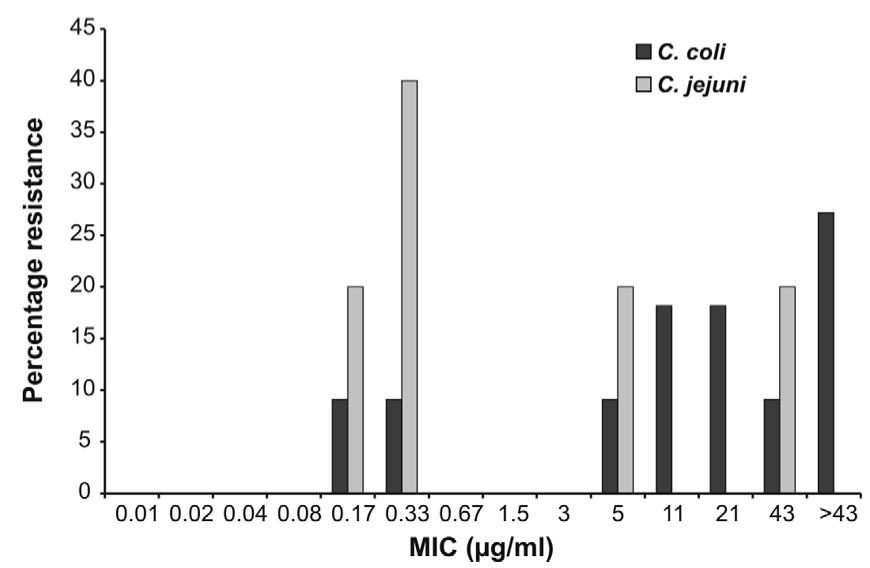

Fig. 2: Percentage distribution of MIC values to the indicated antibiotics of Campylobacter jejuni $(n=5)$ and $C$. coli $(n=11)$ isolated from the Western Cape.

to be the predominant species ${ }^{15}$. Even though the number of farms tested during this investigation was small, the $43 \%$ infected farms was not unusual with C. coli (5 of 6 isolates) predominating in pigs and C. jejuni (23 of 32 isolates) predominating in poultry.

Poultry and pig farms in South Africa have over the years implemented more stringent infection control measures, such as the all-in-all-out system, in-line chlorination of drinking water, restricted access of humans to farms and high levels of hygiene. Furthermore, farm workers may only wear designated protective clothing and are only permitted to work in a specific area ${ }^{20}$. Under these circumstances the possibility of Campylobacter spp. being on a farm is greatly reduced and it is not unreasonable to expect a low prevalence, such as the $7.66 \%$ obtained in this study. In the absence of infected animals, Campylobacter spp. can be introduced by, for example, outerwear of farm workers, transport vehicles, water, food, wild birds and, to a limited extent, rodents.

Furthermore, very few farms in South Africa practice 'thinning out', a procedure where some birds are removed from the flocks at 35 days of age, to allow the remaining birds to grow more rapidly. The crates that are used to remove these excess birds are often heavily contaminated, thus exposing the remaining birds to thermophilic Campylobacter spp. which spread to all of them by the time they are slaughtered $^{30}$.

It is also not surprising that a patchy distribution of Campylobacter spp. was found, as exemplified by those samples from Gauteng where only 2 of the 6 flocks tested were positive. For example, in a study in which poultry in 4 broiler houses were examined, it was found that those in the 1st broiler house to become infected had a low prevalence of Campylobacter spp. but by the time the birds were slaughtered 4 weeks later these bacterial species could not be isolated. This was not the case in the other houses that were suspected to have been infected by workers from the first house later in the grow-out cycle when $100 \%$ of the birds tested at 4 weeks of age had evidence of intestinal colonisation $^{17}$. It has been reported that proper cleaning and disinfection will destroy Campylobacter spp. in houses ${ }^{30}$.

The nature of the samples and the sampling method and preservation of the specimens were similar to those of other studies in which high isolation rates of Campylobacter spp. were obtained. In addition, the use of both a non-selective culture medium with a filter as well as a selective isolation medium such as Skirrow's will ensure an optimal recovery of most strains of the enteric Campylobacter spp. ${ }^{23}$

There are currently no internationally accepted criteria for testing resistance to Campylobacter species, nor are there accepted breakpoint values ${ }^{28}$. The CLSI (2008) considers the agar diffusion test unreliable and recommends the use of either the agar dilution or broth dilution tests. There are, however, no specific breakpoints for this genus. Therefore if a published breakpoint could not be found (Table 2), the clinical breakpoint for an antimicrobial in the same group or for other Gram-negative bacteria was used in this study.

Therapeutic antimicrobials of choice in human patients suffering from lifethreatening campylobacteriosis are initially the macrolides and thereafter the fluoroquinolones and gentamicin ${ }^{11}$. Resistance to these 2 classes of antibiotics in zoonotic Campylobacter species can 
Table 4: Percentage distribution, $\mathrm{MIC}_{50}, \mathrm{MIC}_{90}$ and percentage resistant strains of Campylobacter coli from the Western Cape $(n=11)$.

\begin{tabular}{|c|c|c|c|c|c|c|c|c|c|c|c|c|c|c|c|c|c|}
\hline \multirow[t]{2}{*}{ Antibiotic } & \multirow[t]{2}{*}{$\%$ Resistant } & \multirow[t]{2}{*}{$\begin{array}{l}\mathbf{M I C}_{50} \\
(\mu \mathrm{g} / \mathrm{m} \ell)\end{array}$} & \multirow[t]{2}{*}{$\begin{array}{l}\mathrm{MIC}_{90} \\
(\mu \mathrm{g} / \mathrm{m} \ell)\end{array}$} & \multicolumn{14}{|c|}{$\begin{array}{l}\text { Percentage of isolates at each concentration } \\
\qquad(\mu \mathrm{g} / \mathrm{ml})\end{array}$} \\
\hline & & & & 0.01 & 0.02 & 0.04 & 0.08 & 0.17 & 0.33 & 0.67 & 1.5 & 3 & 5 & 11 & 21 & 43 & $>43$ \\
\hline Chlortetracycline & 45.4 & 5 & 21 & & & 9.1 & & & 9.1 & & & 18.2 & 18.2 & 27.2 & 18.2 & & \\
\hline Doxycycline & 45.4 & 3 & 11 & 9.1 & & 9.1 & 9.1 & 9.1 & 9.1 & & & 9.1 & & 36.3 & 9.1 & & \\
\hline Erythromycin & 72.7 & $>43$ & $>43$ & & & & 9.1 & & 9.1 & & 9.1 & & & & & & 72.7 \\
\hline Fosfomycin & 0 & 11 & 21 & & & & & 18.2 & 9.1 & 9.1 & & & 9.1 & 27.2 & 27.2 & & \\
\hline Lincomycin & 72.6 & 43 & $>43$ & & & & & 9.1 & 9.1 & & & 9.1 & & 9.1 & 9.1 & 27.2 & 27.2 \\
\hline Enrofloxacin & 27.3 & 0.33 & 11 & & 9.1 & 9.1 & 18.2 & 9.1 & 18.2 & & & 9.1 & 9.1 & 18.2 & & & \\
\hline Norfloxacin & 27.3 & 0.33 & 21 & & & & & 9.1 & 54.5 & 9.1 & & & & 9.1 & 9.1 & 9.1 & \\
\hline Tiamulin & 36.4 & 0.33 & 3 & & & & 18.2 & 18.2 & 27.2 & & 9.1 & 18.2 & 9.1 & & & & \\
\hline Tylosin & 27.2 & 21 & $>43$ & & & & & 9.1 & 9.1 & & & & 9.1 & 18.2 & 18.2 & 9.1 & 27.2 \\
\hline
\end{tabular}

The shaded areas indicate the susceptibility range of each antibiotic tested (refer to Table 2 for the breakpoint values)

Table 5: Percentage distribution, $\mathrm{MIC}_{50}, \mathrm{MIC}_{90}$ and percentage resistant strains of Campylobacter jejuni from the Western Cape $(n=5)$.

\begin{tabular}{|c|c|c|c|c|c|c|c|c|c|c|c|c|c|c|c|c|c|}
\hline \multirow[t]{2}{*}{ Antibiotic } & \multirow[t]{2}{*}{$\%$ Resistant } & \multirow[t]{2}{*}{$\begin{array}{l}\text { MIC }_{50} \\
(\mu \mathrm{g} / \mathrm{m} \ell)\end{array}$} & \multirow[t]{2}{*}{$\begin{array}{l}\text { MIC }_{90} \\
(\mu \mathrm{g} / \mathrm{m} \ell)\end{array}$} & \multicolumn{14}{|c|}{$\begin{array}{l}\text { Percentage of isolates at each concentration } \\
\qquad(\mu \mathrm{g} / \mathrm{m} \ell)\end{array}$} \\
\hline & & & & 0.01 & 0.02 & 0.04 & 0.08 & 0.17 & 0.33 & 0.67 & 1.5 & 3 & 5 & 11 & 21 & 43 & $>43$ \\
\hline Chlortetracycline & 80 & 43 & $>43$ & & & & & & 20 & & & & & & 20 & 20 & 40 \\
\hline Doxycycline & 60 & 21 & $>43$ & & & & & & 20 & & & & 20 & & 20 & 20 & 20 \\
\hline Erythromycin & 20 & 0.33 & $>43$ & & & & & & 60 & & & 20 & & & & & 20 \\
\hline Fosfomycin & 20 & 11 & $>43$ & & & & & & 40 & & & & & 20 & & 20 & 20 \\
\hline Lincomycin & 40 & 0.33 & $>43$ & & & & & 20 & 40 & & & & & 20 & & & 20 \\
\hline Enrofloxacin & 40 & 0.33 & 11 & & & & & 20 & 40 & & & & & 40 & & & \\
\hline Norfloxacin & 60 & 11 & $>43$ & & & & & & 40 & & & & & 20 & 20 & & 20 \\
\hline Tiamulin & 40 & 0.33 & 3 & & & & & 20 & 40 & & 20 & 20 & & & & & \\
\hline Tylosin & 0 & 0.33 & 43 & & & & & 20 & 40 & & & & 20 & & & 20 & \\
\hline
\end{tabular}

The shaded areas indicate the susceptibility range of each antibiotic tested (refer to Table 2 for the breakpoint values).

increase hospitalisation rates and the cost of therapy, and decrease the survival rate of patients ${ }^{11}$. Several countries, including Canada and the USA, reported trends of increased resistance of $C$. jejuni to the fluoroquinolones, whereas the prevalence of resistance to the macrolides and tetracyclines has remained static ${ }^{14}$. A surveillance programme for antibiotic resistance in Campylobacter spp. of human origin and similar commissioned surveys for resistance in poultry and pigs in France found that, from 1986 to 1998, the prevalence of resistance of Campylobacter spp. from humans to the fluoroquinolones initially increased, only to progressively decline over the following 5 years. This decline was partially associated with decreased fluoroquinolone resistance in poultry and pigs in that country ${ }^{13}$.

The banning of the incorporation of enrofloxacin in poultry feed in the USA in 2000 by the Food and Drug Administration (FDA) of the USA which was effected in 2005 was a direct consequence of documented evidence showing an increased resistance in disease-causing strains of Campylobacter isolated from humans as well as a $10 \%$ resistance in poultry prod$\mathrm{ucts}^{28,29}$. Since fluoroquinolones, especially enrofloxacin and norfloxacin are used to treat resistant E. coli infection in birds, it would be expected that the same holds true for South Africa. This was true for the few isolates $(50 \%$ resistance to enrofloxacin and $60 \%$ resistance to norfloxacin) from poultry in the Western Cape. None was, however, noted in the poultry isolates from Gauteng, nor from the pig isolates in the Western Cape. The farms tested in Gauteng have a niche market in that they supply certain supermarket chains with untreated birds. A study in KwaZulu-Natal found that resistance to the fluoroquinolones was low $(8 \%)$ but much higher to nalidixic acid ${ }^{6}$. This seems to point to differences in the nature of therapies used in the different provinces. It is known that fluoroquinolone resistance develops rapidly in Campylobacter spp., for, unlike other Gram-negative bacteria, the acquisition of fluoroquinolone resistance in Campylobacter spp. does not require stepwise accumulation of gyrA mutations and overexpression of efflux pumps, but is mainly mediated by single-step point mutations in gyrA in the presence of a constitutively expressed multidrug efflux pump, CmeABC ${ }^{42}$.

In South Africa, tylosin is used extensively by both the poultry and pig industries to treat Mycoplasma infections as well as spirochaete infections in pigs. It is also known to be used in sub-therapeutic doses as a performance enhancer. Therefore it was not surprising that cross-resistance to the parent macrolide erythromycin
(46.35\%) in isolates from the Western Cape was detected. The resistance was higher in C. coli $(72.73 \%)$ than in C. jejuni (20\%). Resistance to tylosin was lower at $27.27 \%$ in C. coli isolates. However, most probably due to the small sample size, these differences only tended toward statistical significance $(P=0.0708$ for erythromycin and $P=0.063$ for tylosin). It was found that $71 \%$ of $C$. coli and only $37 \%$ of C. jejuni isolated from birds fed diets supplemented with tylosin were resistant to erythromycin ${ }^{22}$. A high prevalence of resistance among C. coli isolates from humans and poultry to erythromycin, as well as co-resistance between erythromycin and clindamycin has been reported $^{11,24}$. In our study C. coli was highly resistant to both erythromycin $(72.7 \%)$ and lincomycin $(72.6 \%)$, a lincosamide similar to clindamycin. A binomial pattern of resistance to the macrolides, which divides the bacteria into resistant and susceptible populations, has been reported in C. coli ${ }^{36}$; and occurred in both C. jejuni and C. coli in this study (Fig. 1). The resistance of the Western Cape strains of Campylobacter spp. to tiamulin $(38.2 \%)$ was unexpected as no crossresistance has been reported between the macrolides and pleuromutulins. This could, therefore, be a direct consequence of the use of tiamulin in poultry and pigs in that province. Interestingly the 
Table 6: Percentage distribution of Campylobacter species $(n=22), \mathbf{M I C}_{50}, \mathbf{M I C}_{90}$ and percentage resistant strains from broiler caeca in Gauteng.

\begin{tabular}{|c|c|c|c|c|c|c|c|c|c|c|c|}
\hline \multirow[t]{2}{*}{ Antibiotic } & \multirow[t]{2}{*}{$\%$ Resistance } & \multirow[t]{2}{*}{$\begin{array}{c}\mathrm{MIC}_{50} \\
(\mu \mathrm{g} / \mathrm{m} \ell)\end{array}$} & \multirow[t]{2}{*}{$\begin{array}{l}\mathrm{MIC}_{90} \\
(\mu \mathrm{g} / \mathrm{m} \ell)\end{array}$} & \multicolumn{8}{|c|}{$\begin{array}{l}\text { Percentage of isolates at each concentration } \\
\qquad(\mu \mathrm{g} / \mathrm{m} \ell)\end{array}$} \\
\hline & & & & 0.12 & 0.25 & 0.5 & 1 & 2 & 4 & 8 & $>8$ \\
\hline Ceftiofur & 95.5 & $>8$ & $>8$ & & 4.5 & & & & & 9.1 & 86.4 \\
\hline Florfenicol & 0 & 2 & 4 & & 13.6 & & & 59.1 & 27.3 & & \\
\hline Chlortetracycline & 95.5 & $>8$ & $>8$ & & & & 4.5 & & & & 95.5 \\
\hline Oxytetracycline & 95.4 & $>8$ & $>8$ & & & 4.5 & & & & 13.6 & 81.8 \\
\hline Penicillin & 95.4 & $>8$ & $>8$ & 4.5 & & & & & & 4.5 & 90.9 \\
\hline Ampicillin & 85.5 & 8 & 8 & & 4.5 & & 4.5 & & 4.5 & 81.8 & 4.5 \\
\hline Enrofloxacin & 0 & 0.25 & 0.25 & 45.5 & 50 & 4.5 & & & & & \\
\hline Danofloxacin & 0 & 0.5 & 1 & & 4.5 & 50 & 45.5 & & & & \\
\hline Gentamicin & 0 & 1 & 1 & & & & 100 & & & & \\
\hline Neomycin & 0 & 4 & 4 & & & & & & 100 & & \\
\hline Spectinomycin & 0 & 8 & 8 & & & & & & & 100 & \\
\hline Tylosin & 0 & 1 & 2 & & & 4.5 & 54.5 & 36.4 & 4.5 & & \\
\hline Tulathromycin & 0 & 1 & 2 & & & & 68.2 & 31.8 & & & \\
\hline Tilmicosin & 0 & 4 & 4 & & & & & & 100 & & \\
\hline Tiamulin & 0 & 0.5 & 0.5 & & & 100 & & & & & \\
\hline Lincomycin & 0 & 0.25 & 0.25 & & 100 & & & & & & \\
\hline
\end{tabular}

The shaded areas indicate the susceptibility range of each antibiotic tested (refer to Table 1).

published breakpoint for Campylobacter is $1 \mu \mathrm{g} / \mathrm{m} \ell^{19}$, much lower than what is used for other bacteria where the breakpoint is $32 \mu \mathrm{g} / \mathrm{m} \ell^{7}$.

Tetracyclines are extensively used in both the poultry and pig industries in South Africa, as they are broad-spectrum in activity, cheap and can easily be administered in the food and water. It was, therefore, not surprising to find that $95.5 \%$ of the poultry isolates from Gauteng and $52.7 \%$ (doxycycline) and $62.7 \%$ (chlortetracycline) of the Western Cape isolates were resistant to this class of antimicrobial. A recent study of Campylobacter spp. isolated from broilers and layer hens in KwaZulu Natal also revealed a high level of resistance to the tetracyclines of up to $100 \%$. It must be noted, however, that this study used a breakpoint value of $8 \mu \mathrm{g} / \mathrm{ml}$ and not $4 \mu \mathrm{g} / \mathrm{m} l$. Similar trends have been noted in the United Kingdom ${ }^{31}$ and USA with prevalences of up to $99.5 \%$ in the latter country ${ }^{37}$. This is thought to be due to the easy transfer between bacteria of the conjugative plasmid with the tet $(\mathrm{O})$ gene $^{31}$. Countries such as Iceland, in which tetracycline is rarely used, have negligible levels of resistance $(0.3 \%)^{40}$. This high level of tetracycline resistance is rarely recorded in humans and is most probably due to the fact that tetracyclines are not generally employed as first line therapy but are mainly used to treat vector-borne diseases, such as malaria and tick bite fever, as well as certain skin conditions. Therefore, it is unusual to find that tetracycline resistance occurred in 70 , 72 and $69 \%$ of the Campylobacter spp. from humans in Israel, Spain and Japan respectively ${ }^{28,32}$. Concurrently, in Japan, tetracy- cline resistance was high in food-producing animals ${ }^{28}$.

It is well known that $C$. jejuni produces $\beta$-lactamases that confer resistance to the $\beta$-lactam drugs i.e. amoxicillin and ceftiofur at levels of between 83 to $92 \%{ }^{21}$. This was noted for C. jejuni isolated from Gauteng where $82.4 \%$ and $94.1 \%$ of $C$. jejuni isolates were resistant to amoxicillin and ceftiofur respectively. A study in KwaZulu-Natal recorded up to $100 \%$ resistance to ceftriaxone in layers and broil$\mathrm{ers}^{6}$. Interestingly, isolates from children at the Red Cross Hospital in the Western Cape have also shown an increase in resistance from $3.6 \%$ in 2002 to $24.6 \%$ in $2006^{28}$. Treatment of Campylobacter spp. infections using the $\beta$-lactam drugs is not generally recommended as it is believed that the cell wall of $C$. jejuni is impermeable to these antibiotics ${ }^{21}$.

Worldwide, the resistance of the thermophilic Campylobacter spp. to the aminoglycosides is very low $(<1 \%)$. In this study there was no resistance in the Campylobacter spp. isolated from birds in Gauteng to gentamicin, neomycin and spectinomycin. This was interesting, for although gentamicin is hardly ever used in poultry, both neomycin and spectinomycin are routinely used to treat intestinal disease. Interestingly, a study done in a Swiss abattoir revealed an unusual resistance pattern in that $27.7 \%$ of the C. jejuni were resistant to streptomycin with a very low resistance to erythromycin and fluoroquinolones ${ }^{12}$. However, technical errors may have accounted for the unusually high streptomycin resistance as the disk diffusion test which is considered to give erratic results was used ${ }^{7}$.

Campylobacter spp. isolated from the 2 farms in Gauteng tended to have very similar antimicrobial resistance (AMR) patterns which indicates that possibly there was clonal expansion of the strains on the farms. However, since the resistance was generally low, the clonal nature of the isolates can only be proven by genetic fingerprinting. These bacteria exhibited a significantly higher resistance to tetracyclines $(P<0.0001)$ and a lower resistance to tylosin $(P=0.0262)$, lincomycin/ clindamycin $(P=0.0001)$ and enrofloxacin $(P=0.0392)$ than those originating from the Western Cape and even KwaZulu-Natal ${ }^{6}$. As mentioned above, it is possible that the high-level management and the consumer pressure to cease the treatment of broilers prevented the selection of antimicrobial resistance.

Multi-resistance in both C. jejuni and C. coli has been reported both in human and animal isolates throughout the world. The resistance pattern that was noted in the 4 multiresistant Campylobacter spp., especially to tetracyclines, macrolides and fluoroquinolones, has been recorded elsewhere $\mathrm{i}^{11,13,34}$. It is postulated that efflux pumps either encoded by the Campylobacter-specific cmeABC gene or by as yet unidentified genes are responsible. Efflux pumps usually result in increased resistance to several antibiotics at once as they actively remove antibiotics from the bacterial cytosol ${ }^{34}$.

\section{CONCLUSIONS AND \\ RECOMMENDATIONS}

Several studies, including this one, have shown that antimicrobial resistance of Campylobacter spp. isolated from humans and animals is highly variable both geographically and from year to year ${ }^{28}$. In 
poultry flocks or pig herds, antimicrobial resistance is dependent on the level of disease and antimicrobials used. In this study of only a few poultry and pig farms, resistance to not only the fluoroquinolones and macrolides, but also multi-resistance was found. Therefore, constant vigilance for Campylobacter spp. of public health significance should be maintained through the use of surveillance and the rapid reporting of trends ${ }^{28}$. Economic restrictions have meant that studies in Africa, including South Africa, are done on an ad hoc basis and are few and far between. This is evidenced by the paucity of publications originating from this continent and the fact that this genus was not included in the fledgling South African antimicrobial surveillance programme $\mathrm{e}^{35}$.

It is therefore recommended that surveillance is instituted for Campylobacter spp. originating especially from poultry and pigs in South Africa and that the focus should be fluoroquinolones, macrolides and tetracyclines, to which a high level of resistance was found in this study. The surveillance programme should also include poultry and pigs belonging to small scale farmers as the prevalence of these bacteria and AMR in South Africa is unknown. It is also likely that these animal species will have a high carriage rate of thermophilic Campylobacter species ${ }^{18}$.

It has been shown that on farms on which antibiotics are not used, the levels of antimicrobial resistance, although not absent, tend to be very low ${ }^{15}$. Therefore, producers should be encouraged by legislation or by market pressures to reduce not only the use of therapeutic antimicrobials that are known to be effective against Campylobacter spp. but also that of tylosin as a performance enhancer.

\section{ACKNOWLEDGEMENTS}

The authors wish to thank personnel at the poultry and pork abattoirs for assisting with the collection of samples.

\section{REFERENCES}

1. Aarestrup F M, Nielsen E M, Madsen M, Engberg J 1997 Antimicrobial susceptibility patterns of thermophilic Campylobacter spp. from humans, pigs, cattle, and broilers in Denmark. Antimicrobial Agents and Chemotherapy 41:10: 2244-2250

2. Acha P N, Szyfres B 2003 Campylobacteriosis. In Zoonoses and Communicable Diseases Common to Man and Animals. Bacterioses and Mycoses (3rd edn). Pan American Health Organization, Washington DC: 67-72

3. Allos B M 2001 Campylobacter jejuni infections: update on emerging issues and trends. Clinical Infectious Diseases 32: 12011206

4. Andrews J M, Baquero F, Beltran J M, Canton E, Crokaert F, Gobernado M 1983 International collaborative study on stan- dardization of bacterial sensitivity to fosfomycin. Journal of Antimicrobial Chemotherapy 12: 357-361

5. Avrain L, Humbert F, L'Hospitalier R, Sanders P, Vernozy-Rozand C, Kempf I 2003 Antimicrobial resistance in Campylobacter from broilers: association with production type and antimicrobial use. Veterinary Microbiology 96: 267-276

6. Bester L A, Essack S Y 2008 Prevalence of antibiotic resistance in Campylobacter isolates from commercial poultry suppliers in KwaZulu-Natal, South Africa. Journal of Antimicrobial Chemotherapy 62: 1298-1300

7. Clinical and Laboratory Standards Institute (CLSI) 2008 Performance Standards for Antimicrobial Disk and Dilution Susceptibility Tests for Bacteria Isolated From Animals; Approved Standard. 3rd edn). CLSI document M31-A3

8. Clinical and Laboratory Standards Institute 2002 NCCLS. Performance Standards for Antimicrobial Disk and Dilution Susceptibility Tests for Bacteria Isolated From Animals; Approved Standard. 2nd edn). NCCLS document M31-A2

9. Cui S, Ge B, Zheng J, Meng J 2005 Prevalence and antimicrobial resistance of Campylobacter spp. and Salmonella serovars in organic chickens from Maryland retail stores. Applied and Environmental Microbiology 71: $4108-4111$

10. Department of Antibiotics 2007 VetMIC Camp (version 2) method. National Veterinary Institute. Uppsala. Online at: http:// www.sva.se (accessed 15 January 2008)

11. Engberg J, Aarestrup F M, Taylor D E, Gerner-Schmidt P, Nachamkin I 2001 Quinolone and macrolide resistance in Campylobacter jejuni and C. coli: resistance mechanisms and trends in human isolates. Emerging Infectious Diseases 7: 24-34. Online at: http://www.cdc.gov/ncidod/EID (accessed 27 January 2010)

12. Frediani-Wolf V, Stephan R 2003 Resistance patterns of Campylobacter strains isolated from poultry carcasses in a big Swiss poultry slaughterhouse. International Journal of Food Microbiology 89: 233-240

13. Gallay A, Prouzet-Mauleon V, Kempf I, Lehours P, Labadi L, Camou C, Denis M, De Valk H, Desenclos J-C, Mégraud F 2007 Campylobacter antimicrobial drug resistance among humans, broiler chickens, and pigs, France. Emerging Infectious Diseases 13: February. Online at: www.cdc.gov/eid. (accessed 27 January 2010)

14. Gaudreau C, Huguette G 2003 Antimicrobial resistance of Campylobacter jejuni subsp. jejuni strains isolated from humans in 1998 to 2001 in Montréal, Canada, Antimicrobial Agents and Chemotherapy 47: 2027-2029

15. Gebreyes W A, Thakur S, Morrow W E M 2005 Campylobacter coli: prevalence and antimicrobial resistance in antimicrobialfree (ABF) swine production systems. Journal of Antimicrobial Chemotherapy 56: 765-768

16. Gormley F J, Macrae M, Forbes K J, Ogden I D, Dallas J F, Strachan N J C 2008 Has retail chicken played a role in the decline of human campylobacteriosis? Applied and Environmental Microbiology 74: 383-390

17. Gregory E, Barnhart H B, Dreesen D W, Stern N J, Corn J L, 1997 Epidemiological study of Campylobacter spp. in broilers: source, time of colonization, and prevalence. Avian Diseases 41: 890-898
18. Heuer O E, Pedersen K, Andersen J S, Madsen M2001 Prevalence and antimicrobial susceptibility of thermophilic Campylobacter in organic and conventional broiler flocks. Letters in Applied Microbiology 33: 269-274

19. Islam K M S, Klein U, Burch D G S 2009 The activity and compatibility of the antibiotic tiamulin with other drugs in poultry medicine - A review. Poultry Science 88: 23532359

20. Kapperud G, Skjerve E, Vik L, Hauge K, Lysaker A, Aalmen I, Ostroff S M, Potter M 1993 Epidemiological investigation of risk factors for campylobacter colonization in Norwegian broiler flocks. Epidemiology and Infection 111: 245-255

21. Lachance N, Gaudreau C, Lamothe F, Larivitre L A 1991 Role of the $\beta$-lactamase of Campylobacter jejuni in resistance to $\beta$-lactam agents. Antimicrobial Agents and Chemotherapy 35: 813-818

22. Ladely S R, Harrison MA, Fedorka-Cray P J, Berrang ME, Englen MD, Meinersmann RJ 2007 Development of macrolide-resistant Campylobacter in broilers administered sub-therapeutic or therapeutic concentrations of tylosin. Journal of Food Protection 70: 1945-1951

23. Lastovica A J 2006 Emerging Campylobacter spp.: the tip of the iceberg. Clinical Microbiology Newsletter 28: $49-55$

24. Luangtongkum T, Morishita T Y, Ison A J, Huang S, Mcdermott P F, Zhang Q 2006 Effect of conventional and organic production practices on the prevalence and antimicrobial resistance of Campylobacter spp. in poultry. Applied and Environmental Microbiology 72: 3600-3607

25. Mann H B, Whitney D R 1947 On a test of whether one of two random variables is stochastically larger than the other. Annals of Mathematical Statistics 18: 50-60

26. Manning G, Dowson G D, Bagnall M C, Ahmed H, West M, Newell D G 2003 Multilocus sequence typing for comparison of veterinary and human isolates of Campylobacter jejuni. Applied and Environmental Microbiology 69: 6370-6379

27. Mann-Whitney U. Online at: http://en. wikipedia.org/wiki/Mann\% E2\% $80 \% 93$ Whitney_U (accessed 26 January 2010)

28. Moore J E, Barton MD, Blair IS, Corcoran D, Dooley J S G, Fanning S, Kempf I, Lastovica A J, Lowery C J, Matsuda M, Mcdowell D A, Mcmahon A, Millar B C, Rao J R, Rooney PJ, Seal B S, Snelling W J, Tolba O 2006 The epidemiology of antibiotic resistance in Campylobacter. Microbes and Infection 8: 1955-1966

29. Nelson J M, Chiller T M, Powers J H, Angulo F J 2007 Fluoroquinolone-resistant Campylobacter species and the withdrawal of fluoroquinolones from use in poultry: a public health success story. Clinical Infectious Diseases 44: 977-980

30. Newell D G, Fearnley C 2003 Sources of Campylobacter colonization in broiler chickens. Applied and Environmental Microbiology 69: 4343-4351

31. Piddock L J, Griggs D, Johnson M M, Ricci V, Elviss N C, Williams L K, Jørgensen F, Chisholm S A, Lawson A J, Swift C, Humphrey T J, Owen R J 2008 Persistence of Campylobacter species, strain types, antibiotic resistance and mechanisms of tetracycline resistance in poultry flocks treated with chlortetracycline. Journal of Antimicrobial Chemotherapy 62: 303-315 
32. Prats G, Mirelis B, Llovet T, Munoz C, Miro E, Navarro F 2000 Antibiotic resistance trends in enteropathogenic bacteria isolated in 1985-1987 and 1995-1998 in Barcelona. Antimicrobial Agents and Chemotherapy 44: 1140-1145

33. Prescott J F 1990 Campylobacter. In Carter G R, Cole J R (eds) Diagnostic procedures in veterinary bacteriology and mycology (5th edn). Academic Press, San Diego, CA: $61-75$

34. Randall L P, Ridley A M, Cooles S W, Sharma M, Sayers A R, Pumbwe L, Newell D G, Piddock L J V, Woodward M J 2003 Prevalence of multiple antibiotic resistance in 443 Campylobacter spp. isolated from humans and animals Journal of Antimicrobial Chemotherapy 52: 507-510
35. SANVAD 2007 South African National Veterinary Surveillance and Monitoring Programme for Resistance to Antimicrobial Drugs. University of Pretoria, Pretoria

36. Shin L, Lee Y 2007 Antimicrobial resistance of 114 porcine isolates of Campylobacter coli. International Journal of Food Microbiology 118: 223-227

37. Son I, Englen M D, Berrang M E, FedorkaCray P J, Harrison M A 2007 Antimicrobial resistance of Arcobacter and Campylobacter from broiler carcasses. International Journal of Antimicrobial agents 29: 451-455

38. Songer J G, Post K W 2005 The genera Campylobacter, Helicobacter and Arcobacter, In: Songer J G, Post K W (eds) Veterinary microbiology. Elsevier Saunders, St Louis, MO: 223-231
39. Taylor D E, Courvalin P 1988 Mechanisms of antibiotic resistance in Campylobacter species. Antimicrobial Agents and Chemotherapy 32: 1107-1112

40. Thorsteinsdottir T R, Kristinsson K G, Fridriksdottir V, Gunnarsson E 2008 Antimicrobial resistance of Campylobacter spp. isolated from broiler flocks in Iceland 2001-2005. Microbial Drug Resistance 14: 49-53

41. Van der Walt M L 2004 Campylobacter jejuni infection. In Coetzer J A W, Tustin R C (eds) Infectious diseases of livestock (2nd edn) Vol. 3. Oxford University Press, Cape Town: 1479_ 1483

42. Zhang Q 2008 Campylobacteriosis In Saif Y M (ed.) Diseases of poultry (12th edn). Blackwell Publishing, Ames, IA: 675-689 